ELECTRONIC RESEARCH ANNOUNCEMENTS OF THE AMERICAN MATHEMATICAL SOCIETY

Volume 8, Pages 47-51 (December 19, 2002)

S $1079-6762(02) 00104-\mathrm{X}$

\title{
MAXIMAL REGULARITY FOR PARABOLIC EQUATIONS WITH INHOMOGENEOUS BOUNDARY CONDITIONS IN SOBOLEV SPACES WITH MIXED $L_{p}$-NORM
}

\author{
PETER WEIDEMAIER
}

(Communicated by Michael E. Taylor)

\begin{abstract}
We determine the exact regularity of the trace of a function $u \in$ $L_{q}\left(0, T ; W_{p}^{2}(\Omega)\right) \cap W_{q}^{1}\left(0, T ; L_{p}(\Omega)\right)$ and of the trace of its spatial gradient on $\partial \Omega \times(0, T)$ in the regime $p \leq q$. While for $p=q$ both the spatial and temporal regularity of the traces can be completely characterized by fractional order Sobolev-Slobodetskii spaces, for $p \neq q$ the Lizorkin-Triebel spaces turn out to be necessary for characterizing the sharp temporal regularity.
\end{abstract}

\section{INTRODUCTION}

The space $W_{p, q}^{2,1}\left(\Omega_{T}\right):=L_{q}\left(0, T ; W_{p}^{2}(\Omega)\right) \cap W_{q}^{1}\left(0, T ; L_{p}(\Omega)\right), \Omega_{T}:=\Omega \times(0, T)$, $\Omega \subset \mathbb{R}^{n}$, is often employed in the theory of evolution equations which are of first order in time and second order in space; see von Wahl 15 for parabolic equations, Sohr [12, Iwashita [8] for the Navier-Stokes equation, and Clément and Prüss 3 ] for parabolic Volterra equations. For the heat equation (as model problem) this space corresponds to maximal regularity if the inhomogeneous part in the equation belongs to $L_{q}\left(0, T ; L_{p}(\Omega)\right)$. Results of maximal regularity type have been established under various conditions ([2], [4, 6], [9]), but always for homogeneous boundary conditions or the Cauchy problem. Combining these results with Theorems 2.3 and 2.4 stated below, maximal regularity follows also for problems with inhomogeneous boundary conditions.

\section{Trace theory in the Classical Case $p=q \in(1, \infty)$}

The trace of $u \in W_{p, p}^{2,1}\left(\Omega_{T}\right)$ (space denoted $W_{p}^{2,1}\left(\Omega_{T}\right)$ in [11]) belongs to the space $W_{p}^{2-1 / p,(2-1 / p) / 2}\left(\Gamma_{T}\right)$, where $W_{p}^{\alpha, \beta}\left(\Gamma_{T}\right):=L_{p}\left(0, T ; W_{p}^{\alpha}(\Gamma)\right) \cap W_{p}^{\beta}\left(0, T ; L_{p}(\Gamma)\right)$, $W_{p}^{s}$ denoting the Sobolev-Slobodetskii spaces and $\Gamma_{T}:=\Gamma \times(0, T), \Gamma:=\partial \Omega$. This result is sharp. The analogous result holds for the trace of a spatial derivative $\partial_{j} u, j \in\{1, \ldots, n\}$, which is an element of $W_{p}^{1-1 / p,(1-1 / p) / 2}\left(\Gamma_{T}\right)$. For these results see Ladyzhenskaya, Solonnikov, and Ural'tseva [11, Chapter II, Lemma 3.4] and Grisvard [5, Théorème 4.2]. While the Russian authors used the "method of integral representation", Grisvard applied interpolation theory.

Received by the editors October 16, 2002.

2000 Mathematics Subject Classification. Primary 35K20, 46E35; Secondary 26D99.

Key words and phrases. Maximal regularity, inhomogeneous boundary conditions, trace theory, mixed norm, Lizorkin-Triebel spaces. 


\section{Trace theory in the CASE $1<p \leq q<\infty$}

The present author [16] has shown continuity of the map

$$
\left.W_{p, q}^{2,1}\left(\Omega_{T}\right) \ni u \mapsto u\right|_{\Gamma_{T}} \in L_{q}\left(0, T ; W_{p}^{2-1 / p}(\Gamma)\right) \cap W_{q}^{(2-1 / p) / 2}\left(0, T ; L_{p}(\Gamma)\right),
$$

but had to leave open whether this map is onto. In a retrospective view, this target space was not the optimal one. It turned out that the sharp result is obtained if the regularity of the trace in the time variable is described by the Lizorkin-Triebel space $F_{q, p}^{(2-1 / p) / 2}\left(0, T ; L_{p}(\Gamma)\right)$, whose definition follows.

Definition 2.1. A function $g \in L_{q}\left(0, T ; L_{p}(\Gamma)\right)$ belongs to the class

$$
F_{q, p}^{\beta}\left(0, T ; L_{p}(\Gamma)\right), \beta \in(0,1)
$$

iff

$$
\left(\int_{0}^{T}\left(\int_{0}^{T-t} h^{-1-p \beta}\|g(\cdot, t+h)-g(\cdot, t)\|_{L_{p}(\Gamma)}^{p} d h\right)^{q / p} d t\right)^{1 / q}<\infty .
$$

Remark 2.2. The Lizorkin-Triebel spaces $F_{q, p}^{\beta}(\mathbb{R}, \mathbb{R})$ defined by Fourier analysis have a finite difference characterization of the type occurring in the previous definition (see Triebel [14 2.5.10 Theorem]). This fact motivated us to introduce the Lizorkin-Triebel spaces on the bounded domain $(0, T)$ as above. Since $\beta<1$, one can use first order discrete differences in the definition instead of second order ones, which the reader might have expected.

Let us introduce

$$
W F_{p, q}^{\alpha, \beta}\left(\Gamma_{T}\right):=L_{q}\left(0, T ; W_{p}^{\alpha}(\Gamma)\right) \cap F_{q, p}^{\beta}\left(0, T ; L_{p}(\Gamma)\right)
$$

and endow this space with the norm

$$
\|g\|_{W F_{p, q}^{\alpha, \beta}\left(\Gamma_{T}\right)}=\|g\|_{L_{q}\left(0, T ; W_{p}^{\alpha}(\Gamma)\right)}+|g|_{F_{q, p}^{\beta}\left(0, T ; L_{p}(\Gamma)\right)},
$$

where $|g|_{F_{q, p}^{\beta}\left(0, T ; L_{p}(\Gamma)\right)}$ is given by the integral in (2.1).

We are going to formulate our two main theorems, in which we assume $\Omega \subset \mathbb{R}^{n}$ is an open subset (not necessarily bounded) with a compact boundary of the class $C^{1,1}$ (see [10, 6.2.2 Definition] for details ). Moreover, $\nu$ denotes the vectorfield of outer unit normals on $\Gamma$.

Theorem 2.3. i) For $3 / 2<p \leq q<\infty$ the map $\left.u \longmapsto u\right|_{\Gamma_{T}}$, well-defined for those functions $u \in W_{p, q}^{2,1}\left(\Omega_{T}\right)$ which are continuous w.r.t. $x \in \bar{\Omega}$, has a continuous extension

$$
\gamma_{D}: W_{p, q}^{2,1}\left(\Omega_{T}\right) \longrightarrow W F_{p, q}^{2-1 / p,(2-1 / p) / 2}\left(\Gamma_{T}\right) .
$$

ii) For $3<p \leq q<\infty$ the map $\left.u \longmapsto \nabla u \cdot \nu\right|_{\Gamma_{T}}$, well-defined for those functions $u \in W_{p, q}^{2,1}\left(\Omega_{T}\right)$ which are continuously differentiable w.r.t. $x \in \bar{\Omega}$, has a continuous extension

$$
\gamma_{N}: W_{p, q}^{2,1}\left(\Omega_{T}\right) \longrightarrow W F_{p, q}^{1-1 / p,(1-1 / p) / 2}\left(\Gamma_{T}\right)
$$


Theorem 2.4. Let $1 \leq p \leq q<\infty$. For $k=0,1$ there exist continuous linear operators

$$
Z_{k}: W F_{p, q}^{2-k-1 / p,(2-k-1 / p) / 2}\left(\Gamma_{T}\right) \longrightarrow W_{p, q}^{2,1}\left(\Omega_{T}\right)
$$

such that $\gamma_{D} Z_{0}=I_{0}$ for the $p, q$ as detailed in Theorem 2.3 i) and $\gamma_{N} Z_{1}=I_{1}$ for the $p, q$ as detailed in Theorem 2.3 ii), where $I_{k}$ denotes the identity map in $W F_{p, q}^{2-k-1 / p,(2-k-1 / p) / 2}\left(\Gamma_{T}\right)$.

This latter theorem shows in particular that the trace operators $\gamma_{D}$ and $\gamma_{N}$ from Theorem 2.3 are onto, thus implying that the trace spaces found are sharp. The first theorem is proved (in a half-space situation) by the "method of integral representation" introduced by the Russian school [7]. The full details are given in 21]. The Dirichlet case in Theorem 2.4 is proved in [18. Crucial in the proofs of both theorems are certain weighted Hardy inequalities (see [1] and [20]).

3. Existence Results of MAXimal REgularity In THE SPACE $W_{p, q}^{2,1}\left(\Omega_{T}\right)$ FOR LINEAR PARABOLIC BOUNDARY VALUE PROBLEMS OF SECOND ORDER WITH INHOMOGENEOUS BOUNDARY CONDITIONS

We consider the following problems:

$$
\begin{aligned}
u_{t}-a_{i j}(x, t) \partial_{i} \partial_{j} u+a_{i}(x, t) \partial_{i} u+a_{0}(x, t) u & =f(x, t) & & \text { in } \Omega_{T}, \\
u(x, 0) & =u_{0}(x) & & \text { in } \Omega,
\end{aligned}
$$

with Dirichlet boundary condition

$$
u(\xi, t)=g(\xi, t) \quad \text { on } \Gamma_{T}
$$

or conormal boundary condition

$$
a_{i j}(\xi, t) \nu_{i}(\xi) \partial_{j} u(\xi, t)+b_{0}(\xi, t) u(\xi, t)=g(\xi, t) \quad \text { on } \Gamma_{T} .
$$

For convenience we write $u \in \mathcal{P}_{\mathrm{D}}\left(u_{0}, f, g\right)$ if $u$ solves (3.1), (3.2) with the Dirichlet boundary condition (3.3), and we write $u \in \mathcal{P}_{\mathrm{N}}\left(u_{0}, f, g\right)$ if $u$ solves the same problem with the boundary condition (3.4). We are interested in solutions $u \in$ $W_{p, q}^{2,1}\left(\Omega_{T}\right)$. The theory for $q=p$ is classical; see Ladyzhenskaya, Solonnikov, and Ural'tseva [11, Chapter IV, § 9] for the Dirichlet problem and Solonnikov [13] for the conormal boundary condition. More recently the case $q \neq p$ was treated, e.g., within the theory of analytic semigroups by interpolation methods (see Cannarsa and Vespri [2]) or by vector-valued Calderón-Zygmund theory (see Hieber and Prüss [6] and Krylov [9]). In these papers only homogeneous boundary conditions were considered. Combining these results with our sharp trace results from Section 2, we can incorporate inhomogeneous boundary conditions and prove the following existence theorems:

Theorem 3.1. Consider (3.1) $\rightarrow$ (3.3). Assume that $\Omega$ is a bounded domain in $\mathbb{R}^{n}$ of class $C^{2+\varepsilon}$ for some $\varepsilon>0$ and that 
$\left(\mathrm{A}_{D}\right) \quad\left(a_{i j}(x, t)\right)_{1 \leq i, j \leq n}$ is symmetric and positive definite uniformly in $\bar{\Omega} \times[0, T]$, $a_{i j}(x, t) \in C^{0}\left([0, T], C^{0}(\bar{\Omega})\right), a_{i}(x, t) \in C^{0}\left([0, T], L_{r}(\Omega)\right)$ with $r>n$, $a_{0}(x, t) \in C^{0}\left([0, T], L_{s}(\Omega)\right)$ with $s>\frac{n}{2}$;

$\left(\mathrm{E}_{D}\right) \quad 3 / 2<p \leq q<\infty$;

(F) $\quad f \in L_{q}\left(0, T ; L_{p}(\Omega)\right)$;

$\left(\mathrm{g}_{D}\right) \quad g \in W F_{p, q}^{2-1 / p,(2-1 / p) / 2}\left(\Gamma_{T}\right)$;

(Iv) $u_{0} \in B_{p, q}^{2(1-1 / q)}(\Omega)$;

$\left(\mathrm{C}_{D}\right) u_{0}(\cdot)=g(\cdot, 0)$ on $\Gamma$.

Then $\mathcal{P}_{D}\left(u_{0}, f, g\right)$ has a unique solution $u \in W_{p, q}^{2,1}\left(\Omega_{T}\right)$ and there is a constant $c_{D}^{*}(p, q, T)$ with

$\|u\|_{W_{p}^{2,1}\left(\Omega_{T}\right)} \leq c_{D}^{*} \cdot\left(\left\|u_{0}\right\|_{B_{p, q}^{2(1-1 / q)}(\Omega)}+\|f\|_{L_{q}\left(0, T ; L_{p}(\Omega)\right)}+\|g\|_{W F_{p, q}^{2-1 / p,(2-1 / p) / 2}\left(\Gamma_{T}\right)}\right)$.

In the next theorem $B([0, T], X)$ denotes the bounded $X$-valued functions.

Theorem 3.2. Consider (3.1), (3.2), (3.4). Let $\Omega$ be as in the previous theorem. Let $(\mathrm{F}),(\mathrm{Iv})$ from the previous theorem hold unaltered and assume further that $\left(\mathrm{A}_{N}\right)\left(a_{i j}(x, t)\right)_{1 \leq i, j \leq n}$ is symmetric and positive definite uniformly in $\bar{\Omega} \times[0, T]$, $a_{i j}(x, t) \in C^{0}\left([0, T], C^{\mu}(\bar{\Omega})\right) \cap B\left([0, T], C^{1+\varepsilon}(\Gamma)\right) \cap C^{\alpha}\left([0, T], C^{0}(\Gamma)\right)$, $b_{0} \in B\left([0, T], C^{\mu}(\Gamma)\right) \cap C^{\alpha}\left([0, T], C^{0}(\Gamma)\right)$ for some $\varepsilon>0, \alpha>\frac{1}{2}(1-1 / p)$, $\mu>1-1 / p$, and $a_{i}(x, t), a_{0}(x, t)$ are as specified in the previous theorem;

$\left(\mathrm{E}_{N}\right) \quad 3<p \leq q<\infty$;

$\left(\mathrm{g}_{N}\right) \quad g \in W F_{p, q}^{1-1 / p,(1-1 / p) / 2}\left(\Gamma_{T}\right)$

$\left(\mathrm{C}_{N}\right) a_{i j}(\xi, 0) \nu_{i}(\xi) \partial_{j} u_{0}(\xi)+b_{0}(\xi, 0) u_{0}(\xi)=g(\xi, 0)$ on $\Gamma$.

Then $\mathcal{P}_{N}\left(u_{0}, f, g\right)$ has a unique solution $u \in W_{p, q}^{2,1}\left(\Omega_{T}\right)$ and there exists a constant $c_{N}^{*}(p, q, T)$ with

$\|u\|_{W_{p, q}^{2,1}\left(\Omega_{T}\right)} \leq c_{N}^{*} \cdot\left(\left\|u_{0}\right\|_{B_{p, q}^{2(1-1 / q)}(\Omega)}+\|f\|_{L_{q}\left(0, T ; L_{p}(\Omega)\right)}+\|g\|_{W F_{p, q}^{1-1 / p,(1-1 / p) / 2}\left(\Gamma_{T}\right)}\right)$.

Remark 3.3. a) The conditions on the coefficients as formulated in the last two theorems are those obtained in [19]. We do not claim that they are sharp.

b) The Besov space specified in Theorem [3.1 (Iv) is sharp; see [17] and the literature cited therein.

\section{REFERENCES}

1. Besov O. V., Il'in V. P., Nikol'skii S. M., Integral representations of functions and imbedding theorems, vol. 1, Wiley, New York, 1978. MR 80f:46030a

2. Cannarsa P., Vespri V., On maximal $L^{p}$ regularity for the abstract Cauchy problem, Boll. Unione Mat. Ital. (6) 9-B (1986), 165-175. MR 87g:34066

3. Clément Ph., Prüss J., Global existence for a semilinear parabolic Volterra equation, Math. Z. 209 (1992), 17-26. MR 93a:35085

4. Coulhon Th., Duong X. T., Maximal regularity and kernel bounds: observations on a theorem by Hieber and Prüss, Advances Differential Equations, 5 (2000), 343-368. MR 2001d:34087

5. Grisvard P., Commutativité de deux foncteurs d'interpolation et applications, II, J. Math. Pures Appl. 45 (1966), 207-290. MR 36:4362

6. Hieber M., Prüss J., Heat kernels and maximal $L^{p}-L^{q}$ estimates for parabolic evolution equations, Comm. Partial Differential Equations 22 (1997), 1647-1669. MR 98k:34096 
7. Il'in V. P., Solonnikov V. A., On some properties of differentiable functions of several variables, Transl. AMS 81 (1969), 67-90 (Trudy Mat. Inst. Steklov 66 (1962), 205-226). MR 27:2768

8. Iwashita H., $L_{q}-L_{r}$ estimates for solutions of the nonstationary Stokes equations in an exterior domain and the Navier-Stokes initial value problem in $L_{q}$ spaces, Math. Ann. 285 (1989), 265-288. MR 91d:35167

9. Krylov N. V., On the Calderón-Zygmund theorem with applications to parabolic equations, St. Petersburg Math. J. 13 (2002), 509-526 (Algebra i Analiz 13 (2001), 1-25). MR 2002g:35033

10. Kufner A., John O., Fučik S., Function spaces, Noordhoff Int. Publ., Leyden, 1977. MR 58:2189

11. Ladyzhenskaya O. A., Solonnikov V. A., Ural'tseva N. N., Linear and quasilinear equations of parabolic type, Transl. Math. Monogr. 23 Amer. Math. Soc., Providence, RI, 1968. MR 39:3159b

12. Sohr H., The Navier-Stokes equations, Birkhäuser, Basel, 2001.

13. Solonnikov V. A., A priori estimates for second order parabolic equations, Transl. AMS 65 (1967), 51-137 (Trudy Mat. Inst. Steklov 70 (1964), 133-212). MR 28:5267

14. Triebel H., Theory of Function Spaces, Birkhäuser, Basel, 1983. MR 86j:46026

15. von Wahl W., The equation $u^{\prime}+A(t) u=f$ in a Hilbert space and $L^{p}$-estimates for parabolic equations, J. London Math. Soc. 25 (1982), 483-497. MR 84k:34065

16. Weidemaier P., On the trace theory for functions in Sobolev spaces with mixed $L_{p}$-norm, Czechoslovak Math. J. 44 (1994), 7-20. MR 94m:46062

17. Weidemaier P., On the sharp initial trace of functions with derivatives in $L_{q}\left(0, T ; L_{p}(\Omega)\right)$, Boll. Unione Mat. Ital. 9-B (1995), 321-338. MR 96d:46042

18. Weidemaier P., Existence results in $L_{p}-L_{q}$-spaces for second order parabolic equations with inhomogeneous boundary conditions, In: Amann H. et al., Progress in Partial Differential Equations, Proc. Pont-à-Mousson 1997, Pitman Research Notes in Mathematics 384, Longman, Harlow, UK, 1998, pp. 189-200. MR 99e:35092

19. Weidemaier P., Maximal regularity results in Sobolev spaces with mixed $L_{p}$-norm for linear parabolic equations of second order with inhomogeneous boundary conditions, 1998 (unpublished manuscript).

20. Weidemaier P., Sinnamon G., Perturbed weighted Hardy inequalities, Journal Math. Anal. Appl. 234 (1999), 287-292. MR 2000f:26023

21. Weidemaier P., Vector-valued Lizorkin-Triebel spaces and sharp trace theory for functions in Sobolev spaces with mixed $L_{p}$-norm for parabolic problems, submitted.

Fraunhofer-Institut Kurzzeitdynamik, Eckerstr. 4, D-79104 Freiburg, Germany

E-mail address: weide@emi.fhg.de 